\title{
Reflets
}

Revue ontaroise d'intervention sociale et communautaire

\section{La Collective lesbienne}

\section{Denyse Culligan}

Volume 3, numéro 2, automne 1997

Visibles et Partenaires : Pratiques et recherches féministes

URI : https://id.erudit.org/iderudit/026185ar

DOI : https://doi.org/10.7202/026185ar

Aller au sommaire du numéro

Éditeur(s)

Reflets : Revue ontaroise d'intervention sociale et communautaire

ISSN

1203-4576 (imprimé)

1712-8498 (numérique)

Découvrir la revue

Citer cet article

Culligan, D. (1997). La Collective lesbienne. Reflets, 3(2), 250-254.

https://doi.org/10.7202/026185ar

Tous droits réservés (C) Reflets : Revue ontaroise d'intervention sociale et communautaire, 1997

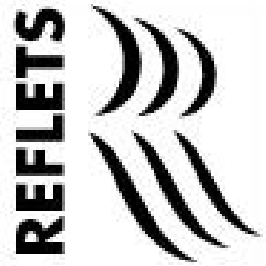

Ce document est protégé par la loi sur le droit d'auteur. L'utilisation des services d'Érudit (y compris la reproduction) est assujettie à sa politique d'utilisation que vous pouvez consulter en ligne.

https://apropos.erudit.org/fr/usagers/politique-dutilisation/ 


\section{La Collective lesbienne}

Denyse Culligan

Coordonnatrice du Comité des femmes francophones du Nord-Ouest de l'Ontario

\section{Introduction}

La discrimination qui s'exerce contre les lesbiennes n'est pas un phénomène nouveau. Pourtant, depuis plusieurs années, nombreuses sont les lesbiennes à s'engager dans le mouvement des femmes de l'Ontario français et à militer en faveur de leurs droits. Cet article proposera donc une brève réflexion sur la question des lesbiennes en Ontario français à partir de l'exemple de la Collective lesbienne, des stratégies et des partenariats développés pour son intégration au mouvement des femmes et des questions brûlantes d'actualité que soulèvent les membres ${ }^{1}$.

\section{Naissance de la Collective lesbienne}

En 1993, lors d'une rencontre de la Table féministe francophone de concertation provinciale de l'Ontario à Ottawa, quelques lesbiennes se sont retrouvées à discuter des politiques et des dossiers de la Table. Lors de ces discussions, elles se sont aperçues que bien que les lesbiennes étaient très actives dans leurs organismes respectifs, elles n'étaient pas représentées formellement comme groupe autonome au sein de la Table. Or, les lesbiennes présentes à cette rencontre souhaitaient une visibilité au sein de la Table comme auprès des divers groupes de femmes et féministes 
francophones de l'Ontario. C'est donc suite à un sondage sur la pertinence de créer un groupe représentatif des lesbiennes qu'est née la Collective lesbienne. Elle s'est donnée comme mandat de créer un réseau afin d'échanger sur les réalités des lesbiennes et de faire de la revendication politique.

\section{Stratégies d'intégration}

C'est un fait généralement admis que le lesbianisme est mal vu dans notre société et que les idées véhiculées à l'égard des lesbiennes sont plus souvent négatives que positives. Par conséquent, l'intégration des lesbiennes dans les organismes communautaires s'effectue souvent «en cachette». Individuellement, les lesbiennes étaient déjà actives comme féministes dans plusieurs dossiers - violence, santé, développement économique, juridico-politique et politique - et avaient acquis une crédibilité, à titre de membres de divers groupes sociaux, pour faire reconnaitre les intérêts des lesbiennes dans tous les dossiers. Cependant, au niveau politique, il fallait qu'elles soient reconnues comme lesbiennes, qu'elles se donnent une visibilité et une voix/voie. En se dotant d'une organisation à leur image et en fondant la Collective lesbienne, les lesbiennes sortent de l'ombre. La Collective a été rapidement reconnue par certains groupes de femmes. Tous les éléments militaient en sa faveur: crédibilité des membres, solidarité et expertises diverses.

Depuis sa fondation, la Collective lesbienne a reçu une première subvention qui lui a permis de publier deux numéros de son bulletin $A u$-delà de l'invisibilité 2 . Par la suite, elle a reçu une autre subvention pour démarrer l'organisation (rencontres, réseautage et concertation sur des dossiers politiques) et pour permettre de faire une analyse critique de la situation des lesbiennes francophones de l'Ontario ${ }^{3}$. Finalement, un troisième organisme subventionnaire a appuyé un projet de recherche sur la violence vécue par les lesbiennes ${ }^{4}$. 
Toutefois, le meilleur exemple de l'intégration des lesbiennes dans le mouvement féministe francophone de l'Ontario s'est effectué au sein de la Table. À l'heure actuelle, plusieurs membres de la Collective occupent des postes stratégiques dans cette organisation; les membres de la Collective sont donc en mesure d'influencer la prise de décisions même si celles-ci ne portent pas exclusivement sur la défense des droits des lesbiennes.

\section{Engagement et partenariats}

Le marrainage et les partenariats que la Collective a établis avec la Table et certains des groupes membres ont joué un rôle important dans la reconnaissance du nouvel organisme. Pourtant, plusieurs questions demeurent sans réponses. Les bailleurs de fonds ont-ils accordé des subventions à la Collective parce que le fait d'être membre de la Table ajoute à sa crédibilité? D'où vient cette popularité? Les lesbiennes sont-elles finalement et véritablement visibles et reconnues? Les dossiers importants pour les lesbiennes sont-ils maintenant à la mode ou est-ce les lesbiennes qui le sont?

Après maintes discussions et réflexions, la Collective est arrivée à la conclusion que sa croissance et sa reconnaissance de la part du mouvement féministe francophone en Ontario sont liées à l'engagement passé et présent de ses membres dans la défense des droits de toutes les femmes. Les membres estiment que la Collective n'aurait jamais obtenu autant de reconnaissance si elle n'avait pas été marrainée par une organisation comme la Table. Les lesbiennes n'auraient jamais atteint cette crédibilité auprès des organismes subventionnaires. Sans vouloir créer une opposition entre les femmes, il est impossible de taire cette vérité. C'est parce que nous sommes féministes d'abord que nous sommes reconnues. Si nous nous étions présentées publiquement que comme représentantes des lesbiennes, aurions-nous eu cette reconnaissance? Nous ne le croyons pas. Précisons. 
Nous n'avions pas encore commencé à revendiquer les droits des lesbiennes que nous nous heurtions déjà un défi particulier. Dans le cadre du projet $A u$ delà de l'invisibilité, nous avons développé un programme de formation en deux volets: la conception d'un atelier pilote et la formation d'animatrices. L'atelier de formation a été conçu dans le but d'aborder deux composantes: sensibiliser les gens à la réalité que vivent les lesbiennes et faciliter l'accès des lesbiennes aux services, aux programmes et aux instances décisionnelles des groupes de femmes. Des équipes d'animatrices, formées d'une femme lesbienne et d'une femme hétérosexuelle, sont actuellement en place. La formation qu'elles offrent vise, en premier lieu, les groupes de femmes et, en second lieu, les groupes mixtes francophones. Toutefois, aucun des groupes de femmes que nous avons rejoints pour offrir cette formation n'a voulu nous recevoir. Est-ce le résultat ou un exemple d'un comportement homophobe? Chose certaine, il existe toujours une peur chez les individus et les groupes d'être associés à des lesbiennes. Appartient-il aux lesbiennes de relever ce défi ou appartient-il aux groupes de femmes ontariennes de surmonter cet obstacle? Comme société, nous devons réfléchir à ces questions et agir car elles sont toujours d'actualité. Il faut éliminer la peur pour être capable de relever ce défi. Nous savons qu'il est possible de travailler collectivement. Les membres de la Collective lesbienne possèdent certaines expertises, les outils et les moyens de sensibiliser la francophonie à leur réalité et ce, dans le respect des différences. Nous avons maintenant besoin de toutes les femmes pour assurer l'intégration des lesbiennes, ce qui ne pourra que renforcer la solidarité, les partenariats et la collaboration qui sont présentement de première importance à la survie de toutes les femmes.

\section{Notes}

1. L'auteure tient à remercier Marie-Luce Garceau pour son aide à la rédaction de cet article.

2. La première subvention a été acccordée par la Direction générale de la condition féminine de l'Ontario. Le bulletin Au-delà de l'invisibilité est publié à chaque trimestre. On peut s'abonner à l'adresse suivante: Collective lesbienne, case postale 79164, 262 
chemin Montréal, Ottawa, Ontario, K1L 1A1.Vous pouvez aussi joindre l'organisme en composant le (613) 745-7505, par télécopieur au (613) 747-8753 ou par courrier électronique à lyne@web.net.

3. La seconde subvention provient du Programme de promotion de la femme de Condition féminine Canada.

4. La troisième subvention a été accordée par l'Office des affaires francophones. 